\title{
Quantum and classical resonant escapes of a strongly driven Josephson junction
}

\author{
H. F. Yu, X. B. Zhu, Z. H. Peng, W. H. Cao, D. J. Cui, Ye Tian, G. H. Chen, D. N. Zheng, \\ X. N. Jing, Li Lu, and S. P. Zhao \\ Beijing National Laboratory for Condensed Matter Physics, Institute of Physics, Chinese Academy of Sciences, \\ Beijing 100190, China \\ Siyuan Han \\ Department of Physics and Astronomy, University of Kansas, Lawrence, Kansas 66045, USA \\ (Received 4 October 2009; revised manuscript received 10 February 2010; published 28 April 2010)
}

\begin{abstract}
The properties of phase escape in a dc superconducting quantum interference device (SQUID) at $25 \mathrm{mK}$, which is well below quantum-to-classical crossover temperature $T_{c r}$, in the presence of strong resonant ac driving have been investigated. The SQUID contains two $\mathrm{Nb} / \mathrm{Al}-\mathrm{AlO}_{x} / \mathrm{Nb}$ tunnel junctions with Josephson inductance much larger than the loop inductance so it can be viewed as a single junction having adjustable critical current. We find that with increasing microwave power $W$ and at certain frequencies $\nu$ and $\nu / 2$, the single primary peak in the switching current distribution, which is the result of macroscopic quantum tunneling of the phase across the junction, first shifts toward lower bias current $I$ and then a resonant peak develops. These results are explained by quantum resonant phase escape involving single and two photons with microwave-suppressed potential barrier. As $W$ further increases, the primary peak gradually disappears and the resonant peak grows into a single one while shifting further to lower $I$. At certain $W$, a second resonant peak appears, which can locate at very low $I$ depending on the value of $\nu$. Analysis based on the classical equation of motion shows that such resonant peak can arise from the resonant escape of the phase particle with extremely large oscillation amplitude resulting from bifurcation of the nonlinear system. Our experimental result and theoretical analysis demonstrate that at $T \ll T_{c r}$, escape of the phase particle could be dominated by classical process, such as dynamical bifurcation of nonlinear systems under strong ac driving.
\end{abstract}

DOI: 10.1103/PhysRevB.81.144518

PACS number(s): 74.50.+r, 05.45.-a, 85.25.Cp, 03.75.Lm

\section{INTRODUCTION}

Devices based on Josephson junctions are not only the key elements for the realization of superconducting qubits ${ }^{1}$ but also testbeds for the study of macroscopic quantum phenomena and nonlinear dynamics. In these studies, response of the device to resonant or near-resonant microwave fields provides insights to understand their behavior. Numerous works have been reported in the literature, such as the demonstration of energy level quantization in current biased junctions $^{2-6}$ and Rabi oscillations in phase qubits. ${ }^{6-8}$ These experiments are usually performed at low temperatures $(\sim 20 \mathrm{mK})$ in relatively weak microwave field. Under such conditions, the junctions behave quantum mechanically and the phase particle may escape from the potential well by macroscopic quantum tunneling (MQT) from ground state and/or via photon-assisted tunneling (PAT) from excited states, depending on the bias current $I$ and the microwave frequency $\nu$. When the switching current distribution $P(I)$ of a current biased junction is measured, MQT from ground state manifests as a peak, which we call the primary peak, centered slightly below the critical current $I_{c}$ of the junction. In the case of the PAT process, a resonant peak at a slightly lower current $I_{\text {res }}$ could be observed in addition to the primary peak in $P(I)$.

The phase escape of a Josephson junction at low temperatures exhibits a number of interesting phenomena as the microwave power $W$ increases, which can be described as follows. (1) As mentioned above, when the frequency $\nu$ matches the bias-current-dependent energy level spacing between the ground and the first excited states at $I_{\text {res }}$ that is close to $I_{c}$ and $W$ is low, the excited-state population $\rho_{1}$ will be enhanced but remains $\ll 1$. This results in a higher escape rate $\Gamma \simeq\left(1-\rho_{1}\right) \Gamma_{0}+\rho_{1} \Gamma_{1}>\Gamma_{0}$ at $I_{\text {res }}$ since $\Gamma_{1} \sim 10^{3} \Gamma_{0}$. Consequently, a resonant peak at $I_{\text {res }}$ appears in $P(I)$. Because $W$ is low, the system's potential and level spacing are essentially unperturbed by microwave field, as reflected in the unchanged position of the primary peak. (2) When $\nu$ corresponds to the level spacing at a resonance current $I_{\text {res }}$ that is well below $I_{c}$ and the microwave field is weak, there will be no resonant peak since $\Gamma_{1}$ is still too low to observe at $I_{\text {res }}$. In this case, as $W$ is increased, the position of the primary peak will move continuously to lower bias currents due to the suppression of the effective potential barrier. ${ }^{9-11}$ This suppression is particularly strong at resonance, leading to a significant increase in $\Gamma_{1}$ and thus a visible resonant peak. (3) If $\nu$ matches a fraction of the level spacing and satisfies $2 \pi \nu m=\omega_{0 n}$, where $\hbar \omega_{0 n}$ is the level spacing between the ground state and the $n$th excited state and $m$ is a small positive integer, the multiphoton PAT process occurs and the corresponding resonant peak appears. (4) When $W$ further increases, the system's nonlinear characteristics such as nonlinear resonance, bifurcation, and chaotic dynamics may emerge.

The resonant peak has also been observed at higher temperatures (e.g., $4.2 \mathrm{~K}$ ) in the classical regime where thermal activation over the top of the barrier dominates. ${ }^{12-15}$ In this regime, when the driving frequency $\nu$ matches the junction's bias-current-dependent plasma frequency $\omega_{p}(I)$, the classical resonance occurs. The oscillation amplitude of the phase particle in the potential well and thus the escape rate can be greatly enhanced. Recently, such process and their classical 
interpretation have been studied in extended temperature range and have received much attention due to a series of works by Grønbech-Jensen and co-workers. ${ }^{16-20}$ These authors demonstrated that previously quantum mechanically explained single- or multiphoton resonance, the effective barrier suppression, and the Rabi oscillation can also be understood from the classical point of view. Interpretations on both the classical and quantum bases are also reported ${ }^{19-22}$ for the results involving multiphoton, multilevel Rabi oscillation, and ac Stark shift at high $W .^{21-24}$ It is found that at high $W$, the predictions of the classical and quantum pictures may converge. ${ }^{22}$ These results indicate that the Josephson junction system can have a dual character, classical and quantum mechanical, when a microwave field is applied.

In this paper, we investigate the switching current distribution and the escape rate of a dc superconducting quantum interference device (SQUID) as a function of microwave field strength at low temperatures. The SQUID contains two $\mathrm{Nb} / \mathrm{Al}-\mathrm{AlO}_{x} / \mathrm{Nb}$ junctions with Josephson inductance much greater than the loop inductance so it behaves as a single junction with a tunable critical current. We found that as microwave power $W$ is increased while frequency $\nu$ is kept constant, the primary peak of $P(I)$ first shifts toward lower bias current and then a resonant peak develops. This result may find a classical explanation ${ }^{11,17,18}$ but here we will show instead that it is also in accordance with the situation (2) discussed above. We further found that the effect of twophoton process on escape rate can be observed in this situation. Moreover, as $W$ further increases, the primary peak will gradually disappear and the resonant peak grows into a single one while shifting continuously to the lower bias current. At even higher $W$, a second resonant peak appears, which could locate at a very low bias current depending on the value of $\nu$. Analysis based on the classical equation of motion indicates that such double-resonant-peak structure with a large separation between the two peaks at high $W$ can originate from the resonant escape of the phase particle having a very large oscillation amplitude resulting from bifurcation of the nonlinear system driven by a strong microwave field. ${ }^{25-27}$

\section{EXPERIMENTAL TECHNIQUES AND RESULTS WITHOUT MICROWAVE RADIATION}

The sample used in this work was a dc SQUID containing two $\mathrm{Nb} / \mathrm{Al}-\mathrm{AlO}_{x} / \mathrm{Nb}$ junctions, which was prepared at Stony Brook using a self-aligned process. ${ }^{28}$ Each junction had a diameter of $1.27 \mu \mathrm{m}$ (corresponding to an area of $1.27 \mu \mathrm{m}^{2}$ ) and the SQUID loop had an area of $220 \mu \mathrm{m}^{2}$. The device's oscilloscope $I-V$ trace at $25 \mathrm{mK}$ is displayed in the inset of Fig. 1. Taking $I_{c} / 2 \sim 1 \mu \mathrm{A}$, we find the Josephson inductance of $330 \mathrm{pH}$ for each junction, which is much larger than the SQUID loop inductance of $\sim 30 \mathrm{pH}$. Hence, the SQUID can be viewed as a single junction having a maximum critical current $I_{c}$.

According to the resistively and capacitively shunted junction (RCSJ) model, ${ }^{29}$ the dynamics of a single junction biased at $I$ can be described by a fictitious phase particle with position $\varphi$, mass $M=C\left(\Phi_{0} / 2 \pi\right)^{2}$, and friction coefficient $1 / R C$ moving in a washboard potential

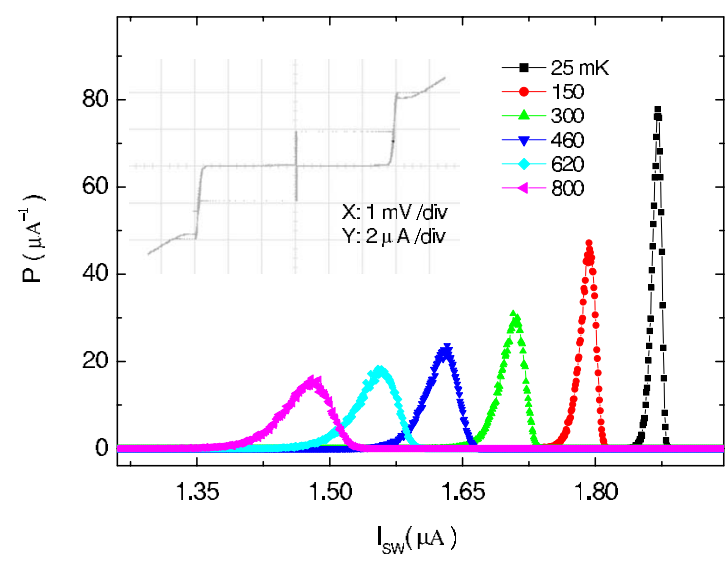

FIG. 1. (Color online) Switching current distribution $P(I)$ at several temperatures between 25 and $800 \mathrm{mK}$ for the sample used in this work. The inset shows the oscilloscope $I-V$ image at $25 \mathrm{mK}$.

$$
U(i, \varphi)=-E_{J}(i \varphi+\cos \varphi),
$$

in which $\Phi_{0}=h / 2 e$ is the flux quantum, $E_{J}=I_{c} \Phi_{0} / 2 \pi$, and $i \equiv I / I_{c}$. $C$ and $R$ are the capacitance and shunt resistance of the junction, respectively. The plasma frequency of the junction is given by $\omega_{p}=\omega_{0}\left(1-i^{2}\right)^{1 / 4}$, where $\omega_{0}=\sqrt{2 \pi I_{c} / C \Phi_{0}}$. For $i<1$, the phase particle may escape from the potential well either by thermal activation (TA) or by macroscopic quantum tunneling, resulting in the junction's switching from the zero to the finite voltage state. Crossover from MQT to TA is known to occur at $T_{c r}=\hbar \omega_{p}\left[\left(1+\alpha^{2}\right)^{1 / 2}-\alpha\right] / 2 \pi k_{B}$, where $\alpha$ $=1 / 2 Q$, and $Q=\omega_{p} R C$ is the quality factor. The escape rate in the TA regime can be found from Kramers' formula ${ }^{30}$

$$
\Gamma_{t}=\frac{\omega_{p}}{2 \pi} a_{t} \exp \left(-\frac{\Delta U}{k_{B} T}\right),
$$

where $a_{t}=4 /\left(\sqrt{1+Q k_{B} T / 1.8 \Delta U}+1\right)^{2}$ is a damping dependent factor and in the MQT regime from ${ }^{31}$

$$
\Gamma_{q}=\frac{\omega_{p}}{2 \pi} a_{q} \exp \left[-\frac{7.2 \Delta U}{\hbar \omega_{p}}\left(1+\frac{0.87}{Q}\right)\right],
$$

where $a_{q} \simeq\left[120 \pi\left(7.2 \Delta U / \hbar \omega_{p}\right)\right]^{1 / 2}$. In the above expressions, the barrier height is given by

$$
\Delta U(i)=2 E_{J}\left[\sqrt{1-i^{2}}-i \arccos (i)\right] .
$$

We measured switching current distribution $P(I)$ using the time-of-flight technique, as described in our previous works. $^{32,33}$ The results at some typical temperatures are shown in Fig. 1. In our experiment, the bias current was ramped up at a constant rate of $d I / d t=0.4 \mathrm{~mA} / \mathrm{s}$ with a repetition rate of $97 \mathrm{~Hz}$. To reduce statistical uncertainty each measured $P(I)$ contained $5 \times 10^{4}$ escape events. The sample was placed in a copper box, which was anchored to the mixing chamber (MXC) of an Oxford Kelvinox MX400 dilution refrigerator. To reduce the effects of external noise on switching dynamics to a negligible level, a trilayer $\mu$-metal shield was used and the circuit was filtered by electromagnetic interference (EMI) filters at room temperature, cryogenic low-pass filters at $1 \mathrm{~K}$ pot, and copper powder microwave filters at MXC temperature. Since noise could 


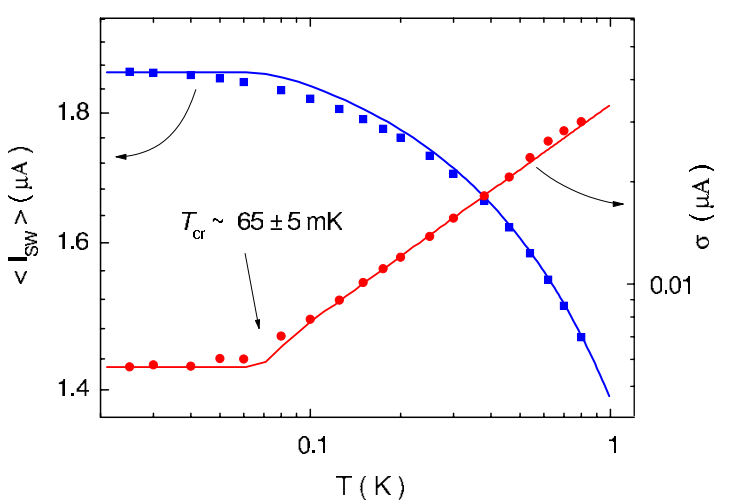

FIG. 2. (Color online) Temperature dependence of the mean $\left\langle I_{s w}\right\rangle$ and standard deviation $\sigma$ of the measured $P(I)$ (symbols). Solid lines are the predictions from TA and MQT theories using the parameters listed in Table I. The experimental MQT-to-TA crossover temperature $T_{c r}=65 \pm 5 \mathrm{mK}$ is indicated.

cause temperature dependence of the width of $P(I)$ to flatten out at $T>T_{c r}$, which could be mistaken as the evidence for MQT, we tested the noise level by applying a flux to the SQUID loop to reduce its critical current to $I_{c}(\Phi) \ll I_{c}(0)$. The result confirmed that noise from the environment and measurement circuitry was negligible down to $25 \mathrm{mK}$. [The data presented in this work were however obtained with maximum critical current $I_{c}=I_{c}(0)$ in the absence of applied magnetic field. The plasma frequency at bias current $\sim 1.865 \mu \mathrm{A}$, which is the position of maximum $P(I)$ at 25 $\mathrm{mK}$ in Fig. 1, is $\omega_{p} \sim 10 \mathrm{GHz}$.]

Figure 1 shows that as temperature decreases, $P(I)$ narrows and moves to higher bias current. In Fig. 2, we show the temperature dependence of the mean $\left(\left\langle I_{s w}\right\rangle\right)$ and width $(\sigma)$ of $P(I)$ (symbols), together with the results calculated from the TA and MQT theories (lines) using the standard conversion between $\Gamma_{t, q}$ and $P(I) .{ }^{34}$ From the fit, the sample's parameters $I_{c}=1.957 \mu \mathrm{A}, C=620 \mathrm{fF}$, and $R=300 \Omega$ were obtained. ${ }^{35}$ In Fig. 2, we can see that in the temperature range between $1 \mathrm{~K}$ and $T_{c r} \simeq 65 \mathrm{mK}, \sigma$ decreases with decreasing temperature indicating that TA is the dominant escape mechanism. In this temperature range, $P(I)$ depends weakly on $C$ and $R$ so $I_{c}$ can be determined by fitting $P(I)$ using the TA theory starting with a reasonable estimate of $C$ and $R$ values. The values of $C$ and $R$ were then determined using $P(I)$ obtained at $T \ll T_{c r}$ because the exponent of MQT rate is dependent on $C$ and $R$. In Table I, we summarize the sample's key parameters. Taking the $P(I)$ peak position at 25 $\mathrm{mK}$ in Fig. 1 again, we find that the crossover temperature calculated from these parameters is $T_{c r}=62.4 \mathrm{mK}$, which is in good agreement with the experimental value indicated in Fig. 2.

TABLE I. Sample parameters.

\begin{tabular}{cccc}
\hline $\begin{array}{c}\text { Junction area } \\
\left(\mu \mathrm{m}^{2}\right)\end{array}$ & $\begin{array}{c}I_{c} \\
(\mu \mathrm{A})\end{array}$ & $\begin{array}{c}C \\
(\mathrm{fF})\end{array}$ & $\begin{array}{c}R \\
(\Omega)\end{array}$ \\
\hline $2 \times 1.27$ & 1.957 & 620 & 300 \\
\hline \hline
\end{tabular}

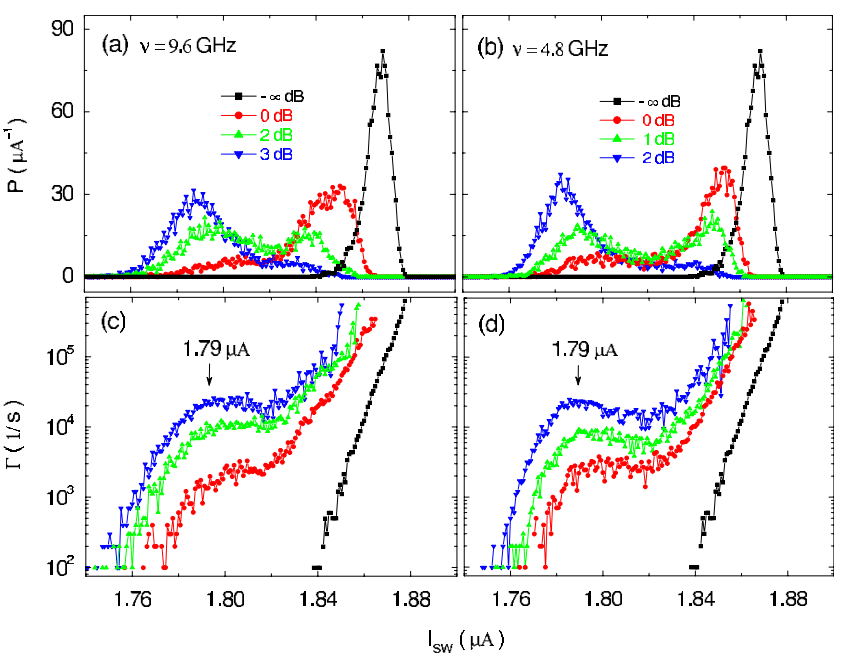

FIG. 3. (Color online) Switching current distribution $P(I)$ and escape rate $\Gamma(I)$ at $25 \mathrm{mK}$ for several microwave powers as indicated. Power levels are relative with the curves where resonant peaks start to appear set to $0 \mathrm{~dB}$, and the two $0 \mathrm{~dB}$ curves in (a) and (b) correspond to -10 and $-37 \mathrm{dBm}$, respectively. Resonant peaks demonstrating resonant quantum phase escape can be clearly seen. [(a) and (c)] $\nu=9.6 \mathrm{GHz}$, single-photon process and [(b) and (d)] $\nu=4.8 \mathrm{GHz}$, two-photon process. The resonance current $I_{\text {res }}$ is indicated by the arrows in (c) and (d).

\section{RESULTS WITH MICROWAVE RADIATION}

Figure 3(a) shows the switching current distribution $P(I)$ at $25 \mathrm{mK}$ in the microwave field with fixed frequency $\nu$ $=9.6 \mathrm{GHz}$ and four different power levels. (In this work, we always use relative power levels by normalizing to the power level at which the resonant peak first appears.) Figure 3(c) shows the corresponding escape rate $\Gamma$. It is seen that as $W$ increases, the primary peak of $P(I)$ shifts to lower bias current and then a resonant peak develops. Further increasing $W$ causes the primary peak to disappear and resonant peak to grow into a single one (Results at higher $W$ are presented in Fig. 4 below). This result, though similar to situation (1) discussed in Sec. I, can be interpreted along a quantummechanical analysis by Fistul et al..$^{9}$ since the primary peak demonstrates a clear leftward shift resulting from effective barrier suppression. ${ }^{36}$

According to the discussion in Ref. 9, the condition $\left(2 \pi \nu / \omega_{0}\right)^{5} \leq \hbar \omega_{0} / E_{J}$ should be satisfied in order for the process described in situation (1) to occur. The physics behind this is that the microwave-induced excited-level population times the escape rate of the level should be large. Taking the junction's parameters in Table I, we find $\left(2 \pi \nu / \omega_{0}\right)^{5} \sim 0.088$ and $\hbar \omega_{0} / E_{J} \sim 0.016$ so the condition is clearly not satisfied. On the other hand, we calculated the energy levels, and the level escape and transition rates of the system using the approach described in Ref. 37 . The total escape rate $\Gamma_{L O}$ was then obtained from the stationary solution of the Master equation as first considered by Larkin and Ovchinnikov. ${ }^{6,37,38}$ We found that for the data without microwave radiation $(-\infty \mathrm{dB})$ in Figs. 3(a) and 3(c), there were three levels in the potential well and the calculated $\Gamma_{L O}$ agreed well with the experimental result. If we assume an unsuppressed potential 


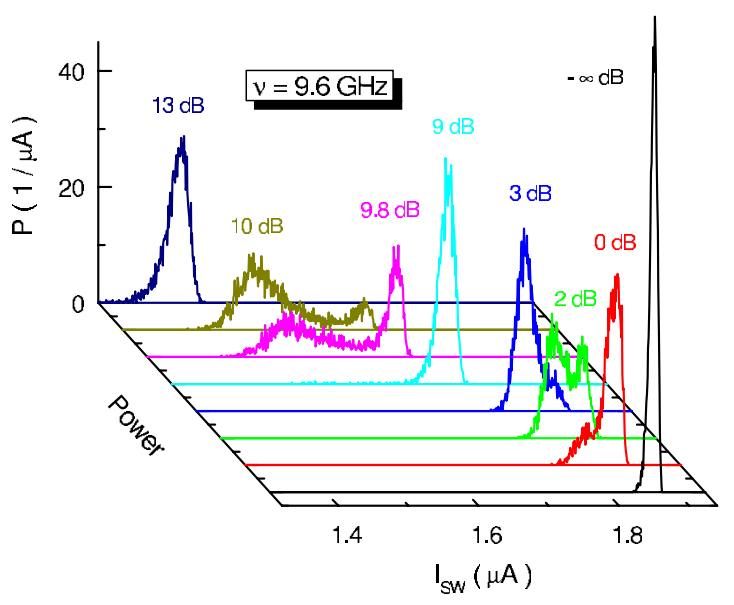

FIG. 4. (Color online) Switching current distribution $P(I)$ at 25 $\mathrm{mK}$ with different relative microwave power $W$. The $0 \mathrm{~dB}$ curve corresponds to a power level of $-10 \mathrm{dBm}$. The microwave frequency is $\nu=9.6 \mathrm{GHz}$. It is seen that as $W$ increases, the doublepeak structure appears and disappears twice. The appearance of the two resonant peaks can be explained by quantum resonant escape and nonlinear classical resonant escape, respectively.

barrier in the microwave field and use $I_{\text {res }}=1.79 \mu \mathrm{A}$ at the resonant peak, we would have six energy levels in the well. In this case, even assuming all population at the first excited level would still lead to $\Gamma_{L O}$ about three to four orders smaller than the measured escape rate. From these results, it is clear that a barrier suppression resulting in a reduction in one or two energy levels in the well is necessary to account for the experimental data in Fig. 3.

The calculated level spacing $\omega_{01} / 2 \pi$ between the ground and first excited states at $I_{r e s}=1.79 \mu \mathrm{A}$, which can also be estimated from $\omega_{01}=\omega_{p}\left(1-5 \hbar \omega_{p} / 36 \Delta U\right)$, is $\sim 9.6 \mathrm{GHz}$, which exactly matches the frequency of the applied microwave. This result demonstrates that the quantum explanation of the resonant phase escape process is applicable. In Figs. $3(\mathrm{~b})$ and 3(d), we show the corresponding results when the microwave frequency is reduced to $\nu=\left(\omega_{01} / 2 \pi\right) / 2$ $=4.8 \mathrm{GHz}$. Similar behavior can be seen, which is consistent with the quantum picture of the resonant phase escape as a result of the two-photon process. ${ }^{39}$

As $W$ increases, the primary peak in Fig. 3 decreases and eventually disappears while the resonant peak grows into a single one. The data taken at $\nu=9.6 \mathrm{GHz}$ are plotted in Fig. 4. The most interesting feature of the data in this figure is that a second resonant peak develops when $W$ is further increased. ${ }^{40}$ Compared with the previous case of weak microwave field, the separation between the second resonant peak and the first resonant peak is much larger than that between the first resonant peak and the primary peak. Furthermore, the separation is found to depend strongly on the microwave frequency $\nu$. In Fig. 5, we show the $W$ dependence of $P(I)$ at $25 \mathrm{mK}$ when $\nu$ is changed to $7 \mathrm{GHz}$ which is largely detuned from the level spacing $\omega_{01} / 2 \pi$ $\simeq 9.6 \mathrm{GHz}$ in the vicinity of the bias current where the primary peak is located. Therefore, resonant peak originated from PAT (e.g., the peaks just below the primary peak in Figs. 3 and 4) would not occur at low microwave power. As

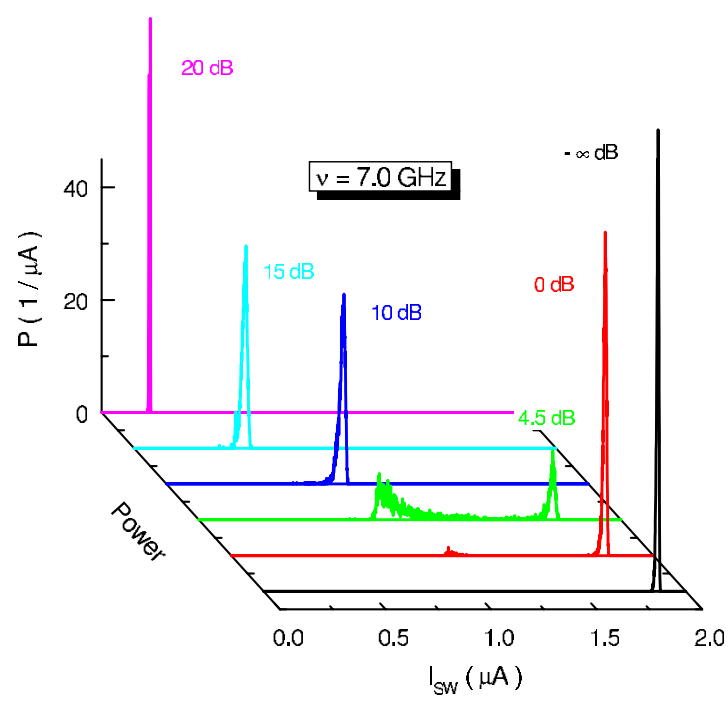

FIG. 5. (Color online) Switching current distribution $P(I)$ at 25 $\mathrm{mK}$ with different relative microwave power $W$. The $0 \mathrm{~dB}$ curve corresponds to a power level of $-15 \mathrm{dBm}$. The microwave frequency is $\nu=7 \mathrm{GHz}$. In contrast to the results in Fig. 4, the doublepeak structure appears and disappears only once. Here the resonant peak results from the nonlinear classical resonant escape of the phase particle.

$W$ increases, the system gradually enters the strong driving regime. The primary peak is observed to shift leftward continuously until a resonant peak at a much lower bias current $\sim 0.85 \mu \mathrm{A}$ develops (note the different horizontal scales of Figs. 4 and 5). Such distinct results could originate from Josephson bifurcation, ${ }^{26,27}$ a phenomenon common to strongly driven nonlinear dynamic systems, as discussed below.

\section{DISCUSSION BASED ON THE CLASSICAL NONLINEAR DYNAMICS}

It should be pointed out that the number of levels in the potential well at the second resonant peak (at $\sim 1.5 \mu \mathrm{A})$ in Fig. 4 and the corresponding peak at $\sim 0.85 \mu \mathrm{A}$ in Fig. 5 would be about 20 and over 50 , respectively. The presence of so many energy levels during resonant escape, provided a dramatic barrier suppression did not occur, necessitates a many-photon process from the quantum-mechanical point of view or motion with extremely large amplitude oscillations from the perspectives of classical dynamics. Since it is not clear whether the treatment of Fistul et al. ${ }^{9}$ is applicable to such case we provide an explanation based on the classical description of dynamic bifurcation in nonlinear systems for the experimental results presented in Figs. 4 and 5. Our analysis shows that the experimental results obtained at high $W$, namely, the double-peak structure with large peak separation, can be caused by escape of the phase particle having very large oscillation amplitude resulting from bifurcation of the nonlinear system driven by strong microwave field. ${ }^{25-27}$

\section{A. Nonlinear bifurcation phenomenon: Bistable oscillations with different amplitudes}

We start with the normalized equation of motion for the phase particle 


$$
\ddot{\varphi}+\frac{1}{Q_{0}} \dot{\varphi}+\sin \varphi=i+i_{m w} \cos (\Omega \tau),
$$

where $\tau=\omega_{0} t, Q_{0}=\omega_{0} R C, i_{m w}=I_{m w} / I_{c}$ is the normalized ac current induced by microwave field, and $\Omega$ is the microwave frequency $\nu$ normalized to $\omega_{0} / 2 \pi$. At low temperatures $(T$ $=25 \mathrm{mK}$ ) and strong microwave field, the effect of noise current $i_{n}$ due to thermal fluctuations on escape rate is negligible and thus omitting $i_{n}$ in Eq. (5) is justified.

Following the analysis of Refs. 9 and 19, we use a monochromatic ansatz for the solution of the unperturbed Eq. (5)

$$
\varphi=\varphi_{0}+\phi(\tau)
$$

with

$$
\varphi_{0}=\arcsin (i) .
$$

Inserting this ansatz into Eq. (5) and expanding $\sin \phi$ and $\cos \phi$ up to the third order yield ${ }^{41}$

$$
\ddot{\phi}+\frac{1}{Q_{0}} \dot{\phi}+\Omega_{1}^{2} \phi+a \phi^{2}+b \phi^{3}=i_{m w} \cos (\Omega \tau),
$$

in which $\Omega_{1}=\left(1-i^{2}\right)^{1 / 4}, a=-i / 2$, and $b=-\sqrt{1-i^{2}} / 6$. When the applied microwave frequency $\Omega$ is close to the resonance, we can write $\Omega=\Omega_{1}+\delta \Omega$, where $\delta \Omega / \Omega \ll 1$, and make the single-mode approximation

$$
\phi=A \sin (\Omega \tau+\eta),
$$

where $\eta$ is a phase constant and $A$ is amplitude of the driven oscillation. Inserting this into Eq. (8), one obtains the following relation ${ }^{25}$

$$
A^{2}\left(\delta \Omega-\chi A^{2}\right)^{2}+\frac{A^{2}}{4 Q_{0}^{2}}=\frac{i_{m w}^{2}}{4 \Omega_{1}^{2}},
$$

where $\chi=\left(3 b / 8 \Omega_{1}-5 a^{2} / 12 \Omega_{1}^{3}\right)$.

From Eq. (10), the amplitude $A$ as a function of microwave power $W \propto i_{m w}^{2}$ and bias current $i$ can be obtained since $\delta \Omega, \chi, Q_{0}$, and $\Omega_{1}$ only depend on $i$ and/or $W$ when microwave frequency $\Omega$ is constant. Thus, we have

$$
A=A(i, W) .
$$

In Fig. 6, we present the calculated $A(i, W)$ for three indicated bias current $i$ at $\nu=9.6 \mathrm{GHz}$ (solid lines) using the parameters listed in Table I. The results show clearly the familiar Josephson bifurcation phenomenon. ${ }^{26,27}$ Namely, as $W \propto i_{m w}^{2}$ increases, the system can be driven into a regime where two dynamic states with different amplitudes of oscillation $A_{2}>A_{1}$ exist, as shown schematically in Fig. 7. In Fig. 6 , one can see that in the range of $i_{m w}^{2}$ considered bifurcation does not occur at bias current $I=1.9 \mu$ A which is slightly below $I_{c}(i \approx 0.971)$. As $I$ decreases, it starts to develop. For lower bias current, threshold for bifurcation shifts to higher $W$ which is accompanied by a greater amplitude $A_{2}$ at the threshold while $A_{1}$ has a slow variation (the solid lines in Fig. 6). The dashed line in Fig. 6 is the result obtained using $I=1.3 \mu \mathrm{A}$ and $\nu=7.0 \mathrm{GHz}$. In this case, $A_{2}$ is significantly larger when compared to the case of same $I$ but higher driving frequency $\nu=9.6 \mathrm{GHz}$. This result will be shown to be

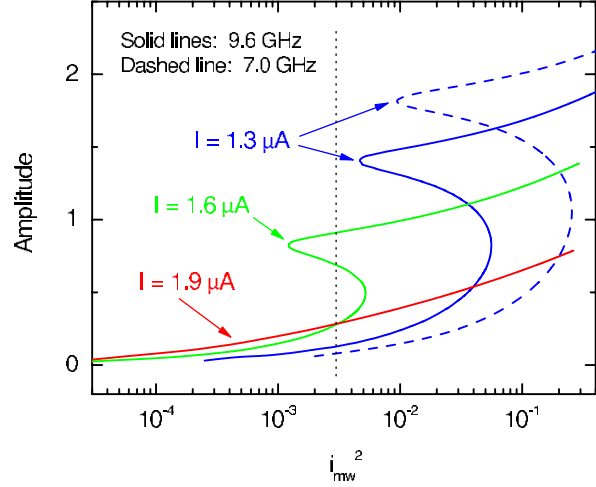

FIG. 6. (Color online) Calculated oscillation amplitude versus $i_{m w}^{2}(\propto$ microwave power $W)$ of the phase particle for different bias current $I$ and frequency $\nu$. The parameters in Table I are used. The results show the familiar bifurcation at low $I$ and high $W$ with two oscillation amplitudes $A_{2}>A_{1}$ (the middle one being unstable for a given curve).

consistent with and can be used to explain the changes from Fig. 4 to Fig. 5 when $\nu$ decreases.

\section{B. Resonant phase escape from bifurcated oscillation with the larger amplitude: Explanation for the results at $\nu=9.6 \mathrm{GHz}$}

We now show that the presence of $A_{2}$ resulting from bifurcation at high microwave power can qualitatively explain the double-peak structure with large peak separation as presented in Figs. 4 and 5. We first consider the four curves with relative power levels of 9, 9.8, 10, and $13 \mathrm{~dB}$ in Fig. 4 with applied microwave frequency $\nu=9.6 \mathrm{GHz}$. In this case, all peaks are located at relatively high bias current, so from the results presented in Fig. 6, the maximum $A_{2}$ is seen to be around 1.5, which makes the approximation used in deriving Eq. (8) appropriate. ${ }^{41}$

Since our measurement was performed at $25 \mathrm{mK}$, escape of the phase particle from the potential via thermal activation is negligible. Hence we consider a classical resonantly oscillating particle and its MQT escape from the potential well. In general, the particle can escape from both $A_{1}$ and $A_{2}$ states,

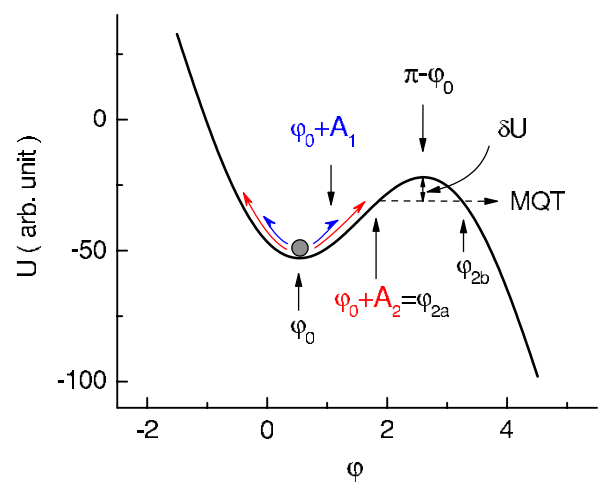

FIG. 7. (Color online) Washboard potential $U(\varphi)$ of Eq. (1) with various phase values, remaining barrier height $\delta U$, and MQT process indicated. 
thus the MQT rate, in the absence of the barrier suppression, can be calculated using the WKB method from

$$
\begin{aligned}
\Gamma_{\mathrm{WKB}}= & p_{1} \Gamma_{A_{1}}+p_{2} \Gamma_{A_{2}} \simeq p_{1} \nu \exp \left(-2 S_{1} / \hbar\right) \\
& +p_{2} \nu \exp \left(-2 S_{2} / \hbar\right) \simeq p_{2} \nu \exp \left(-2 S_{2} / \hbar\right),
\end{aligned}
$$

where $p_{1,2}$ are the probabilities of the phase particle in the $A_{1}$ and $A_{2}$ states $\left(p_{1}+p_{2}=1\right)$ and $S_{1,2}$ are the corresponding path integral across the remaining tunnel barriers. Because $S_{2}$ $\ll S_{1}\left(A_{2} \gg A_{1}\right)$ tunneling rate from the $A_{1}$ state is exponentially smaller and the escape is dominated by the latter. In an explicit form, we can write down the action for the phase particle in the $A_{2}$ state as

$$
S_{2}=\int_{\varphi_{2 a}}^{\varphi_{2 b}} \sqrt{U(i, \varphi)-U\left(i, \varphi_{2 a}\right)} d \varphi,
$$

in which $U$ is given by Eq. (1). The turning point at the outer wall of the potential $\varphi_{2 a}=\varphi_{0}+A_{2}$, the point of escape $\varphi_{2 b}$, and the corresponding path of MQT, when the phase particle is in the $A_{2}$ state, are illustrated in Fig. 7.

For the present experimental data, we usually have a deep potential well $\Delta U$ [see Eq. (4), for bias current well below $I_{c}$ ] and the remaining barrier height $\delta U$ (see Fig. 7) for the particle having $A_{2}$ oscillation amplitude is comparatively small. In this case, it is straightforward to show that Eq. (12) can be well approximated by

$$
\Gamma_{\mathrm{WKB}} \simeq p_{2} \nu \exp \left[-2 \pi \delta U / \hbar \omega_{p}\right],
$$

where $\delta U$ is given by

$$
\delta U(i, W)=U\left(i, \pi-\varphi_{0}\right)-U\left[i, \varphi_{0}+A_{2}(i, W)\right] .
$$

Various phase locations in Eq. (15) are indicated in Fig. 7. In Fig. 8(a), we show the tunneling exponent $2 \pi \delta U / \hbar \omega_{p}$ of Eq. (14) versus $I$ for $\nu=9.6 \mathrm{GHz}$ as $i_{m w}^{2}$ is increased from curve 1 to curve 4 . Notice that for each curve there exists a nearly vertical part, shown as dashed lines, which corresponds to the sudden emergence of the $A_{2}$ state. This can be understood by considering the case for $i_{m w}^{2}=3 \times 10^{-3}$ as indicated by the vertical dotted line in Fig. 6 . At $I=1.3 \mu \mathrm{A}$, only the smaller amplitude $A_{1}$ state exists. The amplitude $A_{1}$ would increase as $I$ increases. At a bias current $1.3 \mu \mathrm{A}<I<1.6 \mu \mathrm{A}$, the much larger amplitude $A_{2}$ state emerges which results in the sudden decrease in $\delta U(i)$ and thus a much larger tunneling rate. In Fig. 8(a), it can be seen that the curve 1 shows a monotonic dependence on I. Starting from the curve 2, however, a local minimum develops. This behavior can, in principle, lead to the double-peak structure in $P(I)$ as $i_{m w}^{2}$ is increased.

To compare with $\Gamma_{q}$, which is the MQT rate in the absence of microwave radiation, we plot the exponent $7.2 \Delta U\left(i_{p}\right) / \hbar \omega_{p}\left(i_{p}\right)$ in $\Gamma_{q}$ [see Eq. (3) considering $Q \sim 10$ ] as a dotted line in Fig. 8(a), where $i_{p}$ corresponds to the position of the primary peak. Notice that the line sits well above the wide shoulder part of the solid curves resulting from the $A_{2}$ state of oscillation. Since tunneling rate depends exponentially on $\delta U / \hbar \omega_{p}$ our data imply $p_{2} \sim \exp \left[2 \pi \delta U / \hbar \omega_{p}\right.$ $\left.-7.2 \Delta U\left(i_{p}\right) / \hbar \omega_{p}\left(i_{p}\right)\right] \ll 1$. Namely, the particle spends more time in the $A_{1}$ state than in the $A_{2}$ state. $^{42}$
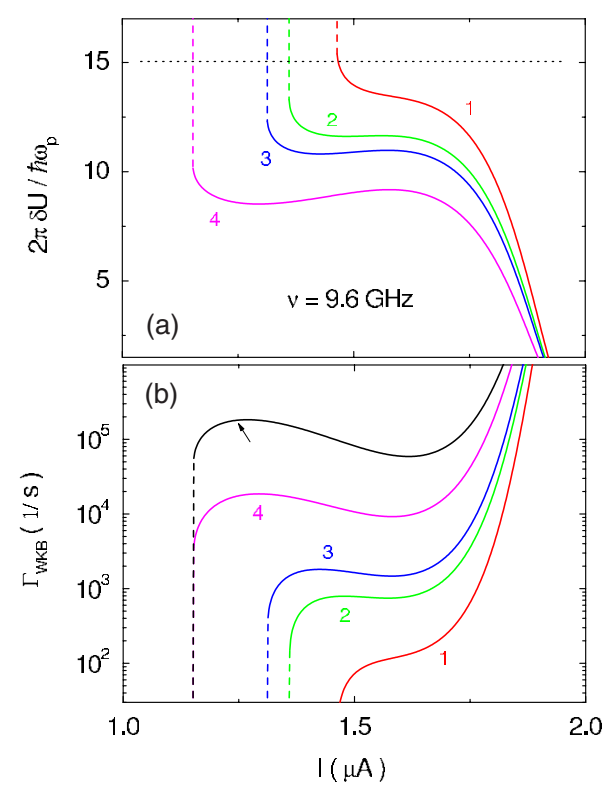

FIG. 8. (Color online) (a) Calculated $2 \pi \delta U / \hbar \omega_{p}$ in Eq. (14) versus $I$ for the microwave frequency $\nu=9.6 \mathrm{GHz}$. Curves labeled $1-4$ have $i_{m w}^{2}=2.5 \times 10^{-3}, 3.7 \times 10^{-3}, 4.3 \times 10^{-3}$, and $6.5 \times 10^{-3}$. The parameters in Table I are used in the calculations. The dotted line is the exponent $7.2 \Delta U\left(i_{p}\right) / \hbar \omega_{p}\left(i_{p}\right)$ in Eq. (3) where $i_{p}$ is the value corresponding to the primary peak without microwave radiation. (b) MQT rate calculated from Eq. (12) and (14) using $p_{2}$ $=10^{-2}$ and the same $\nu$ and $i_{m w}^{2}$ parameters from curves $1-4$ in (a). The line pointed by an arrow has different $\nu=9.4 \mathrm{GHz}$ and $i_{m w}^{2}=6.8 \times 10^{-3}$.

In Fig. 8(b), we show $\Gamma_{\text {WKB }}$ calculated from Eq. (12) and (14) using $p_{2}=10^{-2}$ and the same set of parameters as those in Fig. 8(a). The choice of $p_{2}$ around $10^{-2}$ brings the escape rate $\Gamma_{\mathrm{WKB}}$ for the system in the $A_{2}$ state of oscillation into the experimentally observable range of $10^{2}-10^{6} \mathrm{~s}^{-1}$ in Figs. $3(\mathrm{c})$ and $3(\mathrm{~d})$, which is determined by the bias-current sweeping rate of $d I / d t=0.4 \mathrm{~mA} / \mathrm{s}$. It should be noticed that although the calculated bias-current dependence of $\Gamma_{\mathrm{WKB}}$ in Fig. 8(b) looks similar to the data presented in Figs. 3(c) and $3(\mathrm{~d})$ as the microwave power increases, the nonmonotonic part of $\Gamma_{\mathrm{WKB}}(I)$ covers a much larger range of bias current. Furthermore, unlike the data in Figs. 3(c) and 3(d), the hump feature in Fig. 8(b), which lead to the double peak $P(I)$ moves much faster to lower currents with increasing microwave power. These distinct difference led us to conclude that the double peak distributions in Fig. 3 were originated from photon-assisted tunneling rather than the dynamic bifurcation under strong ac driving described above.

However, as the intensity of ac field is increased the dominant escape mechanism changes from PAT to Josephson bifurcation. Namely, the onset of $A_{2}$ state results in doublepeak distributions with large interpeak separation. In Fig. 9, the corresponding switching current distributions $P(I)$ converted from the $\Gamma_{\mathrm{WKB}}(I)$ presented in Fig. 8(b) are plotted. The existence of the double-peak structure (curves labeled 2 and 3) can be clearly seen. Comparing the results with the experimental data in Fig. 4 (namely, the curves with relative powers of 9, 9.8, 10, and $13 \mathrm{~dB}$ ), we can see that the shapes of $P(I)$ are quite similar and the applied power ratio between 


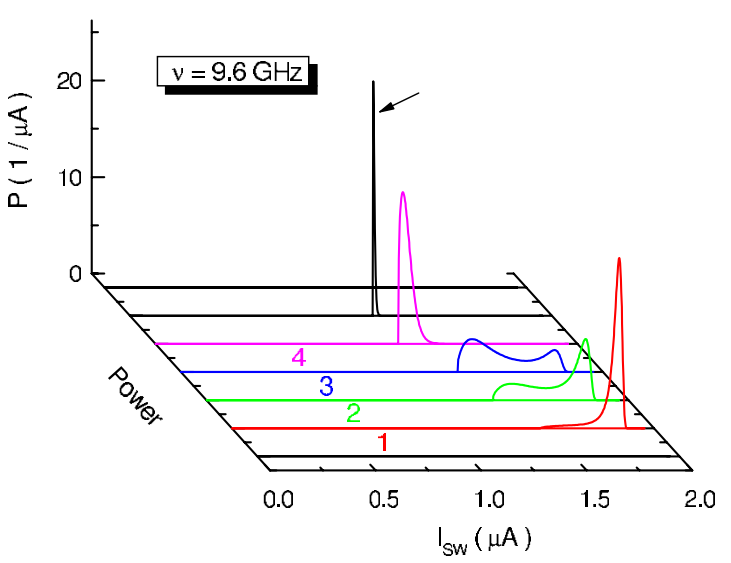

FIG. 9. (Color online) Switching current distributions $P(I)$ converted from $\Gamma_{\mathrm{WKB}}$ in Fig. 8(b). Curve labels have one-to-one correspondence with those in Fig. 8. The data give a qualitative explanation for the development of the second resonant peak in Fig. 4 (curves with relative powers of 9, 9.8, 10, and $13 \mathrm{~dB}$ ). The curve pointed by an arrow is converted from that with arrow in Fig. 8(b) and is displayed with its magnitude divided by 5 .

curve 4 and curve 1 is $\sim 2.5$, also similar to the data displayed in Fig. 4.

\section{Results at $\nu=7.0 \mathrm{GHz}$ and further discussions}

The results of $9.6 \mathrm{GHz}$ microwave radiation suggest that the simple classical model provides a reasonably good qualitative explanation for the reappearance of the double-peak structure in Fig. 4 as ac driving is intensified. Such explanation is expected to work also for the data in Fig. 5 with 7-GHz microwave radiation. Unfortunately, analysis based on Eq. (8) does not work well for the $\nu=7 \mathrm{GHz}$ data since the resonant peak appears below $1 \mu \mathrm{A}$ (see Fig. 5). In this case, we can see from the dashed line in Fig. 6 that $A_{2}$ becomes significantly greater than $\pi / 2$, which makes the expansion of the $\sin \phi$ and $\cos \phi$ terms in obtaining Eq. (8) inapplicable. ${ }^{41}$

Comparing the experimental results in Figs. 4 and 5, there are two clear changes in the bifurcation-induced resonant peaks as the microwave frequency $\nu$ decreases. One is the increase in the interpeak separation of the double-peak distribution and the other is the rapid narrowing of the resonant peak width. The first change can be easily understood from Fig. 6 that for fixed bias current and power, $A_{2}$ is larger when $\nu$ is lower (compare the solid and dashed lines at $I$ $=1.3 \mu \mathrm{A}$ ). This directly leads to the increase in the interpeak separation for smaller $\nu$. It is important to note that such trend is basically opposite to that reported in the previous work where $W \propto i_{m w}^{2}$ is smaller and bifurcation is not involved. ${ }^{17,18}$

The rapid narrowing of the peak can also be explained by our model. To demonstrate this, we plot in Fig. 8(b) the result with $\nu=9.4 \mathrm{GHz}$ (the top curve indicated by the arrow), a value smaller than $9.6 \mathrm{GHz}$ used in the figure but not far away so that the approximation used for Eq. (8) is still valid. We have raised $i_{m w}^{2} \propto W$ slightly so that the vertical part of the curve sits at the same bias current as that of curve 4 .
We can see that the calculated $\Gamma_{\text {WKB }}$ increases quickly, roughly by 1 order of magnitude when $\nu$ is decreased only by 0.2 GHz. Such increase would influence the peak width significantly since, unlike the usual case such as those depicted in Figs. 3(c) and 3(d) where escape rate changes continuously, the bifurcation-related escape rate versus dc bias current makes a sudden jump (dashed parts of the curves in the figure) when $A_{2}$ appears. While the rate of escape from the smaller $A_{1}$ amplitude oscillation is negligible, it increases rapidly when the $A_{2}$ oscillation appears, which results in switching to occur in a very small range of bias current and therefore a much narrower resonant peak. Apparently, the lower the $\nu$ is, the higher level the escape rate will suddenly jump to, and the narrower the resonant peak will be. The curve pointed by the arrow in Fig. 9 is the resulting distribution that appears considerably sharper than curve 4 in the same figure.

It should be pointed out that from our simple model the "primary" and "resonant" peaks in Fig. 9 have different meanings as conventionally understood from the data such as those in Fig. 3. According to the discussion on the results presented in Fig. 8, all peaks in Fig. 9 are caused by tunneling from the $A_{2}$ state of the resonantly driven phase particle. The double-peak structure is a result of combined effect of nontrivial bias-current dependence of the potential well depth $U\left(i, \pi-\varphi_{0}\right)-U\left(i, \varphi_{0}\right)$ and the amplitude $A_{2}(i, W)$ at a given $W$. According to Eqs. (1) and (11), both quantities decrease as $i$ increases but at different rates. This gives rise to the local minima and humps in Fig. 8, and the resonant peak in Fig. 9.

We note that quantitatively the experimental data and calculation based on Josephson bifurcation do not agree well, thus further improvements of the model are necessary. Among them, including higher-order terms in the expansion of $\sin \phi$ and $\cos \phi$ should be considered for in order to describe the data taken at $\nu=7.0 \mathrm{GHz} .^{41} \mathrm{We}$ emphasize that Josephson bifurcation is used to explain the appearance of the second resonant peak in Fig. 4 for data taken at 9.8 and $10 \mathrm{~dB}$. The double-peak structure at much weaker ac driving, from about $0-3 \mathrm{~dB}$, is due to the quantum resonant-phase escape process accompanied by barrier suppression, 9,10 which is not considered in the present model.

\section{SUMMARY}

We presented a systematic study of resonant phase escape in tilted washboard potential of a Josephson junction (a dc SQUID with small loop inductance) at $25 \mathrm{mK}$ in the presence of strong ac driving (microwave radiation). The device was well characterized using the TA and MQT theories in the absence of the microwave field. For weak ac driving $i_{m w}$ $\ll 1$, we observed results that are interpreted by quantum resonant escape of the phase involving single- and twophoton absorptions with a suppressed potential barrier. At larger $i_{m w}$, a second resonant peak, which is well separated from the first one, would appear. The peak could locate at very low bias current depending on the power and frequency of the microwave applied. We proposed a model based on the classical equation of motion to interpret the data. Our results 
indicated that at large $i_{m w}$, the phase particle enters a bistable state due to bifurcation of the nonlinear system and the oscillation state with larger amplitude leads to the resonant peaks in the switching current distributions locating far below the system's critical current. These results are useful for the further studies of the nonlinear response of Josephson junctions to strong ac driving and for the detection of the qubit quantum states using a dc SQUID. In addition, the results and analysis showed that for junctions with strong ac driving, even at $T \ll T_{c r}$, the quantum-to-classical crossover temperature, escape of the phase particle could be dominated by classical processes. Our work therefore provides further evidence of the dual character, classical and quantum me- chanical, of the Josephson junction system at $T \ll T_{c r}$ in an ac driving field.

\section{ACKNOWLEDGMENTS}

We thank V. Patel, W. Chen, and J. E. Lukens for providing us with the $\mathrm{Nb}$ samples used in this work. The work at the Institute of Physics was supported by the National Natural Science Foundation of China (Grant Nos. 10534060 and No. 10874231) and the Ministry of Science and Technology of China (Grant Nos. 2006CB601007, No. 2006CB921107, and No. 2009CB929102). S. Han was supported in part by NSF under Grant No. DMR-0325551.
${ }^{1}$ Y. Makhlin, G. Schon, and A. Shnirman, Rev. Mod. Phys. 73, 357 (2001)

${ }^{2}$ J. M. Martinis, M. H. Devoret, and J. Clarke, Phys. Rev. Lett. 55, 1543 (1985).

${ }^{3}$ J. Clarke, A. N. Cleland, M. H. Devoret, D. Esteve, and J. M. Martinis, Science 239, 992 (1988).

${ }^{4}$ A. Wallraff, T. Duty, A. Lukashenko, and A. V. Ustinov, Phys. Rev. Lett. 90, 037003 (2003)

${ }^{5}$ T. Bauch, T. Lindström, F. Tafuri, G. Rotoli, P. Delsing, T. Claeson, and F. Lombardi, Science 311, 57 (2006).

${ }^{6}$ H. Xu, A. J. Berkley, M. A. Gubrud, R. C. Ramous, J. R. Anderson, C. J. lobb, and F. C. Wellstood, IEEE Trans. Appl. Supercond. 13, 956 (2003).

${ }^{7}$ Y. Yu, S. Han, X. Chu, S. Chu, and Z. Wang, Science 296, 889 (2002).

${ }^{8}$ J. M. Martinis, S. Nam, J. Aumentado, and C. Urbina, Phys. Rev. Lett. 89, 117901 (2002).

${ }^{9}$ M. V. Fistul, A. Wallraff, and A. V. Ustinov, Phys. Rev. B 68, 060504(R) (2003).

${ }^{10}$ M. V. Fistul and A. V. Ustinov, Phys. Rev. B 63, 024508 (2000).

${ }^{11}$ Sun Guozhu, Wang Yiwen, Cao Junyu, Chen Jian, Ji Zhengming, Kang Lin, Xu Weiwei, Yu Yang, Han Siyuan, and Wu Peiheng, Phys. Rev. B 77, 104531 (2008).

${ }^{12}$ M. H. Devoret, J. M. Martinis, D. Esteve, and J. Clarke, Phys. Rev. Lett. 53, 1260 (1984).

${ }^{13}$ B. Mao and S. Han, IEEE Trans. Appl. Supercond. 17, 94 (2007).

${ }^{14}$ T. Fonseca and P. Grigolini, Phys. Rev. A 33, 1122 (1986).

${ }^{15}$ G. Rotoli, T. Bauch, T. Lindstrom, D. Stornaiuolo, F. Tafuri, and F. Lombardi, Phys. Rev. B 75, 144501 (2007).

${ }^{16}$ N. Grønbech-Jensen and M. Cirillo, Phys. Rev. B 70, 214507 (2004).

${ }^{17}$ N. Grønbech-Jensen, M. G. Castellano, F. Chiarello, M. Cirillo, C. Cosmelli, L. V. Filippenko, R. Russo, and G. Torrioli, Phys. Rev. Lett. 93, 107002 (2004).

${ }^{18}$ N. Grønbech-Jensen, M. G. Castellano, F. Chiarello, M. Cirillo, C. Cosmelli, C. Cosmelli, V. Merlo, R. Russo, and G. Torrioli, in Quantum Computing: Solid State Systems, edited by B. Ruggiero, P. Delsing, C. Granata, Y. Paskin, and P. Silvestrini (Springer, New York, 2006), pp. 111-119.

${ }^{19}$ N. Grønbech-Jensen and M. Cirillo, Phys. Rev. Lett. 95, 067001 (2005).
${ }^{20}$ J. E. Marchese, M. Cirillo, and N. Grønbech-Jensen, Phys. Rev. B 73, 174507 (2006).

${ }^{21}$ F. W. Strauch, S. K. Dutta, Hanhee Paik, T. A. Palomaki, K. Mitra, B. K. Cooper, R. M. Lewis, J. R. Anderson, A. J. Dragt, C. J. Lobb, and F. C. Wellstood, IEEE Trans. Appl. Supercond. 17, 105 (2007).

${ }^{22}$ J. Claudon, A. Zazunov, F. W. J. Hekking, and O. Buisson, Phys. Rev. B 78, 184503 (2008).

${ }^{23}$ J. Claudon, F. Balestro, F. W. J. Hekking, and O. Buisson, Phys. Rev. Lett. 93, 187003 (2004).

${ }^{24}$ D. I. Schuster, A. Wallraff, A. Blais, L. Frunzio, R. S. Huang, J. Majer, S. M. Girvin, and R. J. Schoelkopf, Phys. Rev. Lett. 94, 123602 (2005).

${ }^{25}$ L. D. Landau and E. M. Lifshitz, Mechanics (Pergamon Press, New York, 1976).

${ }^{26}$ I. Siddiqi, R. Vijay, F. Pierre, C. M. Wilson, L. Frunzio, M. Metcalfe, C. Rigetti, R. J. Schoelkopf, M. H. Devoret, D. Vion, and D. Esteve, Phys. Rev. Lett. 94, 027005 (2005).

${ }^{27}$ V. E. Manucharyan, E. Boaknin, M. Metcalfe, R. Vijay, I. Siddiqi, and M. Devoret, Phys. Rev. B 76, 014524 (2007).

${ }^{28}$ W. Chen, V. Patel, and J. E. Lukens, Microelectron. Eng. 73-74, 767 (2004).

${ }^{29}$ W. C. Stewart, Appl. Phys. Lett. 12, 277 (1968); D. E. McCumber, J. Appl. Phys. 39, 3113 (1968).

${ }^{30}$ H. A. Kramers, Physica (Utrecht) 7, 284 (1940).

${ }^{31}$ A. O. Caldeira and A. J. Leggett, Phys. Rev. Lett. 46, 211 (1981).

${ }^{32}$ S.-X. Li, W. Qiu, S. Han, Y. F. Wei, X. B. Zhu, C. Z. Gu, S. P. Zhao, and H. B. Wang, Phys. Rev. Lett. 99, 037002 (2007).

${ }^{33}$ D. J. Cui, H. F. Yu, Z. H. Peng, X. B. Zhu, Ye Tian, G. H. Chen, D. H. Lin, C. Z. Gu, D. N. Zheng, X. N. Jing, Li Lu, and S. P. Zhao, Supercond. Sci. Technol. 21, 125019 (2008).

${ }^{34}$ T. A. Fulton and L. N. Dunkleberger, Phys. Rev. B 9, 4760 (1974).

${ }^{35} \mathrm{C}$ was mainly contributed by a designed parallel capacitance in the device.

${ }^{36}$ We point out that classical analysis should also work though quantum-mechanical approach is used here. See Refs. 17, 11, and 18 for the resonant peak development and Refs. 16 and 18 for the leftward shift of the primary peak.

${ }^{37}$ P. Kopietz and S. Chakravarty, Phys. Rev. B 38, 97 (1988). 
${ }^{38}$ A. I. Larkin and Yu. N. Ovchinnikov, Zh. Eksp. Teor. Fiz. 91, 318 (1986) [Sov. Phys. JETP 64, 185 (1987)].

${ }^{39}$ Resonant escape at $4.8 \mathrm{GHz}$ involving two photons in Fig. 3(b) is expected to require a larger driving power than single photon escape at $9.6 \mathrm{GHz}$ in (a). However, the power levels at the output port of the microwave generator were -37 and $-10 \mathrm{dBm}$ for the two $0 \mathrm{~dB}$ curves in Figs. 3(b) and 3(a), respectively, due to the different attenuations at different frequencies of the measurement system. Our measurement showed that the loss along the coaxial line from microwave generator to sample box at 4.8 $\mathrm{GHz}$ was about $10 \mathrm{~dB}$ less than that at $9.6 \mathrm{GHz}$. Furthermore, microwave was coupled to the junction via an electrical dipole antenna. The coupling between the antenna and the junction was weak and strongly frequency dependent so the final microwave power coupled to the junction for the two-photon process at 4.8 $\mathrm{GHz}$ was still larger than that for the single-photon process at
9.6 GHz. Similar results were observed, for example, in J. Lisenfeld, Ph.D. thesis, Friedrich-Alexander-Universität Erlangen-Nürnberg, 2003.

${ }^{40}$ Heating effect induced by microwave field was negligible in our experiment. If we consider the ac current magnitude to be less than $I_{c}$, the maximum heating power is about $0.6 \mathrm{nW}$. This value is much smaller than that generated by junction's switching to the normal state $(\sim 6 \mathrm{nW})$, which already shows negligible influence since the experimental $T_{c r}$ agrees well with the theory.

${ }^{41}$ Such expansion is strict as $\phi \rightarrow 0$, and is reasonable when $\phi$ $<\pi / 2 \sim 1.5$. From the results in Fig. 6 , the treatment can be safely used for the $9.6 \mathrm{GHz}$ data in Fig. 4, but can be inaccurate for those in Fig. 5 at lower microwave frequency.

${ }^{42}$ In the corresponding quantum picture, a particle excited to an energy level near the barrier top by multiphoton process has a smaller probability to MQT out of the potential well than to decay into lower energy levels in the well. 